\title{
Re-Framing historischer Grenzlandschaften. Das Beispiel Drachensee
}

\author{
Patrick Reitinger ${ }^{1} \mathbb{D} \cdot$ Lukáš Novotný ${ }^{\mathbb{D}}$
}

Online publiziert: 12 . Februar 2020

(c) Der/die Autor(en) 2020

\section{Zusammenfassung}

Der vorliegende Beitrag beschäftigt sich mit der Frage, wie in ländlich-peripheren Grenzregionen produktiv mit transnationalem geschichtlichem Erbe umgegangen werden kann. Durch die besondere historische Entwicklung der bayerisch-tschechischen Grenzregion als geografische Außengrenze während des Kalten Kriegs und aufgrund der Belastungen zahlreicher Transformationen im 20. Jahrhundert suchen die ländlichen Kommunen in Bayern und der Tschechischen Republik nach alternativen Ansätzen der grenzüberschreitenden Regionalentwicklung. Am Beispiel des Drachensees in der bayerischen Stadt Furth im Wald wird aufgezeigt, wie durch eine gezielte Neurahmung historischer Grenzlandschaften Impulse für eine touristische Erschließung schrumpfender Regionen entstehen können.

Schlüsselwörter Ländliche Entwicklung · Grenzüberschreitende Zusammenarbeit · Historisches Erbe · Kulturtourismus · Historische Landschaften

\begin{abstract}
This article deals with the question of how transnational historical heritage can be handled productively in rural-peripheral border regions. Due to the special historical development of the Bavarian-Czech border region as a geographical external border during the Cold War and the burdens of numerous transformations in the twentieth century, rural communities in Bavaria and the Czech Republic are looking for alternative approaches to cross-border regional development. The example of the Dragon Lake in the Bavarian town of Furth im Wald illustrates how the targeted reframing of historical border landscapes can generate impulses for the touristic development of shrinking regions.
\end{abstract}

Keywords Rural development · Cross-border cooperation $\cdot$ Historical heritage $\cdot$ Cultural tourism $\cdot$ Historical landscapes

\section{Transnationale Kooperation in ländlichen Grenzregionen}

Ein Fokus der deutschsprachigen geografischen Forschung zu Grenzregionen liegt gegenwärtig vor allem auf urbanen Räumen und Großregionen (Bechtold et al. 2019). Dies kann darauf zurückgeführt werden, dass sich ein Großteil

Patrick Reitinger, M.A.

patrick.reitinger@uni-bamberg.de

doc. Dr. phil. Lukáš Novotný, M.A.

lukas.novotny@ujep.cz

1 Professur für Historische Geographie, Institut für Geographie, Otto-Friedrich-Universität Bamberg, Am Kranen 12, 96047 Bamberg, Deutschland

2 Philosophische Fakultät, Institut für Politikwissenschaften, Univerzita J. E. Purkyně v Ústí nad Labem, Pasteurova 13, 40096 Ustí nad Labem, Tschechien des Grenzverlaufs der Bundesrepublik Deutschland entlang weniger oder kaum ländlich geprägter Kreise und Kommunen erstreckt (Küpper 2016).

Eine Ausnahme stellt die Grenzregion Bayerns zur Tschechischen Republik dar. Diese ist im Vergleich zu anderen Räumen durch eine besondere Ländlichkeit geprägt (ebd.). Dazu kommt eine wichtige historische Komponente, die sich auf die Entwicklung der geografischen Grenzraumforschung in dieser Region auswirkt: Gemeinsam mit der deutsch-polnischen Grenze schließen die deutsch-tschechischen Gebiete an Staaten des ehemaligen Einflussgebiets der Sowjetunion an. Die Trennung durch den Ost-WestKonflikt und der erst verhältnismäßig späte Beitritt Polens und der Tschechischen Republik zur Europäischen Union im Jahr 2004 lassen im Vergleich zu den deutschen Grenzgebieten nach Westeuropa erst sehr junge transnationale Kooperationen und in der Folge entsprechende grenzüberschreitende Forschungen zu. 
Für die bayerisch-tschechischen Beziehungen kann diese Zusammenarbeit erst seit den beginnenden 2010er-Jahren intensiver beobachtet werden. Vor allem für die politischen Entscheidungsträger in München und Prag ergab sich seitdem ein erhöhter Forschungsbedarf zur grenzüberschreitenden Regionalentwicklung, auf den in den letzten Jahren durch einige wenige Einzelstudien reagiert wurde (Grontmij 2015; Chilla und Weidinger 2014).

Für die geografische Forschung zur Grenzregion kann dabei Ähnliches festgestellt werden, was für die bayerischtschechischen Beziehungen im Allgemeinen gilt: Die Untersuchungsregion ist durch die zentralen Ereignisse der nationalsozialistischen Invasion in die damalige Tschechoslowakei 1938/1939, den 2. Weltkrieg und die nach 1945 erfolgten Vertreibungen deutschsprachiger Bevölkerung aus der böhmischen Grenzregion sowie den dann folgenden Kalten Krieg geprägt. Die politischen Verantwortlichen in Bayern und Tschechien versuchen seit einigen Jahren, neben diesem gemeinsamen historischen Erbe neue Wege des Dialogs und der Zusammenarbeit zu finden. Diese geschichtlichen Transformationen des 20. Jahrhunderts wirken sich aber auch auf die wissenschaftlichen Fragestellungen aus, denn in der Forschung zur bayerischtschechischen Grenzregion können die historischen Voraussetzungen des Untersuchungsraums nicht ausgeblendet werden.

Neben den historischen Grundlagen verbinden darüber hinaus auch andere Faktoren Ostbayern und Westböhmen. Beide Regionen sind durch sehr ländliche Strukturen und eine besonders periphere Lage gekennzeichnet, die sich in einem kontinuierlichen Rückgang von Bevölkerung und Infrastruktur in den vergangenen 30 Jahren widerspiegelt. Ausgangsthese dieser Studie ist es, dass es vor allem solche schrumpfenden Regionen sind, die durch ihre schwierige geografische und sozioökonomische Lage ganz besonders motiviert werden, bei zentralen Anliegen der ländlichen Entwicklung über die Grenze hinweg zu kooperieren. Erst die grenzüberschreitende Herangehensweise an diese Herausforderungen bietet neue Chancen für eine nachhaltige Entwicklung dieser Regionen (Havlíček et al. 2018; Svensson 2015). Gerade in historisch so aufgeladenen Kontexten ist es aber die gemeinsame und trennende Geschichte, die den grenzüberschreitenden Kooperationen immer wieder im Wege steht.

Die Kommunen im Grenzgebiet entwickeln neue Strategien, wie sie einerseits das historische Erbe der Region würdigen, andererseits aber auch darüber hinaus neue Wege der transnationalen Regionalentwicklung gehen können. Der Drachensee in der Oberpfälzer Stadt Furth im Wald ist ein Beispiel dafür, wie durch eine gezielte Neurahmung historischer Grenzlandschaften geschichtlich-kulturelle Aspekte mit neuen touristischen Ansätzen verbunden werden. Diese Verknüpfungen von Geschichte und Gegenwart an konkre- ten geografischen Orten sollen Wachstumsimpulse für die schrumpfende Grenzregion liefern. Der vorliegende Beitrag untersucht beispielhaft dieses gezielte Re-Framing einer historischen Grenzlandschaft durch die transnationale $\mathrm{Zu}$ sammenarbeit bayerischer und tschechischer Kommunen.

\section{Re-Framing historischer Grenzlandschaften}

Re-Framing schließt an das Verständnis der Place-Frames in Anlehnung an Deborah Martin an (Martin 2003). Über Place-Frames versuchen soziale Organisationen verschiedene Menschen miteinander zu vereinen und für spezielle ortsbezogene Aufgaben zu sensibilisieren. Im Gegensatz zum Place-Making, also der Herstellung von Orten, legt das Place-Framing einen Fokus auf eher diskursive Praktiken. Die Organisationen versuchen, eine bestimmte (oft pejorativ konnotierte) Zustandsbeschreibung diskursiv so zu rahmen, dass konkrete Handlungsvorschläge und Aktionen zur Veränderung des Zustands durch eine Organisation zu rechtfertigen sind.

Diese Frames können in 3 Dimensionen ausgeprägt sein. Die motivationale Dimension verfolgt das Ziel, Menschen zum aktiven Engagement an einem bestimmten Ort zu bewegen. Dies wird hauptsächlich durch Emotionalisierungen und kollektive Betroffenheit erzeugt, um eine besondere Schutzwürdigkeit hervorzuheben oder Szenarien der Bedrohung aufzuzeigen (z.B. „Wir müssen aktiv werden, damit unser Ort nicht ausstirbt"). Die diagnostische Dimension versucht ein Bewusstsein für die Situation aus der Perspektive der Rahmenden zu schaffen. Strategien sind dabei die besondere Hervorhebung von quantifizierten Daten, um mögliche Probleme greifbarer zu machen (z. B. „Unsere Kommune hat den höchsten Anteil an Touristen in der Region, darauf können wir aufbauen“). Die prognostische Dimension verfolgt das Ziel, eine Organisation bekannter zu machen und die Zustimmung zu den Zielen der Organisation zu erhöhen. Dies erfolgt über die Entwicklung und Darstellung konkreter Handlungsprogramme, um identifizierte Probleme lösen zu können (z. B. ,Wir setzen uns für eine Stärkung des Ortskerns ein, um die Lebensqualität im Dorfinneren auszubauen").

Mit dem Konzept des Re-Framings soll die zeitliche Dimension von Place-Frames betont werden. Wir gehen davon aus, dass Re-Framing dann Anwendung findet, wenn in einem gewissen zeitlichen Abstand zu einem bereits vollzogenen Framingprozess eine Evaluation des Framings stattfindet und daraufhin bisherige Strategien verändert werden, dabei aber kein absoluter Bruch mit der Vergangenheit erfolgt. Diese Neurahmung schafft also eine diskursive Brücke zwischen dem Vorher und dem Zukünftigen. Ziel des Re-Framings ist die Verarbeitung und Reaktion auf historische Entwicklungen für künftige Diskursstränge. 
Verfolgt wird dabei eine Strategie, historische Narrationen fortzuführen, diese aber gezielt durch Innovationskonzepte zu erweitern. Es wird sozusagen durch das Re-Framing von Orten und Landschaften eine Brücke von der Vergangenheit in die Zukunft gelegt. Dieses Re-Framing kann je nach Zielsetzung wieder motivational, diagnostisch oder prognostisch sein, wobei ein Wechsel der Dimension im Vergleich zum ursprünglichen Framingprozess möglich ist.

Re-Framing kann von Organisationen - in Erweiterung von Martins schließen wir auch kommunale Verbände in unsere Perspektive ein und beschränken uns nicht nur auf zivilgesellschaftliche Akteure - auf einzelne Orte oder ganze Landschaften angewendet werden. Es bietet sich besonders in solchen Landschaftskontexten an, die durch sehr starke und gefestigte historische Narrationen die Handlungsmöglichkeiten der Akteure einschränken. In einer Grenzregion wie in Ostbayern oder Westböhmen kann dieses Re-Framing zum Einsatz kommen, wenn Diskurse über vergangene historische Entwicklungen so wirkmächtig sind, dass sie für künftige Strategien nicht unberücksichtigt bleiben können, weil eine solche Nichtbeachtung immer automatisch auch Widerstand erzeugen würde.

\section{Historisches Erbe als Hindernis und Chance}

Grenzüberschreitende Kooperationen sind im Kontext des europäischen Integrationsprozesses schon seit vielen Jahrzehnten fester Bestandteil der europäischen Strukturförderpolitik. Bereits seit 1958 ist mit der Gründung einer deutsch-niederländischen Europaregion mit Sitz in Gronau das Konzept erprobt (Fricke 2014; Church und Reid 1996). Diese langjährigen Erfahrungen in der Praxis transnationaler Zusammenarbeit können aus historischen Gründen für die bayerisch-tschechische Grenzregion nicht angenommen werden.

Ostbayern und Westböhmen sind seit dem Ende des 1. Weltkriegs und der Gründung der damaligen Tschechoslowakischen Republik mit einer Grenzsituation konfrontiert, die eine Kooperation im Kontext der zahlreichen Transformationsprozesse des 20. Jahrhunderts nur schwer möglich machte. Die Zeit des Kalten Kriegs und die unmittelbare Lage am Eisernen Vorhang ließen die Beziehungen zwischen beiden Regionen gegen Null gehen. Diese Ausgangslage wird bei den regionalen Akteuren in den vergangenen 30 Jahren behutsam und kontinuierlich aufgearbeitet. Vor allem die politischen Entscheidungsträger sind dabei ständig auf der Suche nach sachlichen Themen, die das Gespräch über die Grenze hinweg möglich machen, ohne die schwierigen Themen der Vergangenheit aus nationalsozialistischer Gewaltherrschaft, Vertreibung deutschsprachiger Bevölkerung nach dem 2. Weltkrieg, dem Rückbau großer Teile des ehemaligen Sudetenlands und der anschließen- den staatssozialistischen Zeit in den Kern des Dialogs zu rücken.

Wenn von diesen historischen Belastungen abgesehen wird, die in Bayern und Tschechien bis heute zum Teil zu unterschiedlichen Interpretationen führen, ist in der besonderen geografischen Struktur der Region auf beiden Seiten der Grenze eine zentrale Gemeinsamkeit zu finden. Die grenzüberschreitende Zusammenarbeit erfolgt grundsätzlich über die Kooperation verschiedener Akteure, die auf unterschiedliche Art in die Prozesse eingebunden sind, um an einem gemeinsamen Ziel - der Überwindung struktureller Nachteile der ländlichen Peripherie - zu arbeiten. Dieser Ansatz ist bereits im Europäischen Rahmenübereinkommen über die grenzüberschreitende Zusammenarbeit von Gebietskörperschaften angelegt. Das im Jahr 1980 unterzeichnete Abkommen verpflichtet die teilnehmenden Staaten in Art. 1, „die grenzüberschreitende Zusammenarbeit zwischen Gebietskörperschaften in ihrem eigenen Zuständigkeitsbereich und den Gebietskörperschaften im Zuständigkeitsbereich anderer Vertragsparteien zu erleichtern und zu fördern" (Council of Europe 1980; Terlouw 2012). Dadurch, dass mehr als ein Drittel der Bürgerinnen und Bürger in der Europäischen Union in Grenzregionen lebt und arbeitet, liegt ein besonderer Akzent auf diesen Räumen. Grenze ist damit fester Bestandteil des Alltags für einen nennenswerten Anteil der europäischen Bevölkerung (Zimmerbauer 2011). Durch den Rahmen der europäischen territorialen Zusammenarbeit sollen nicht nur die physisch-materiellen Grenzen in Form von Grenzübergängen und Schlagbäumen beseitigt, sondern auch die kommunikativ konstruierten und imaginären Grenzen im Lebensalltag der Menschen abgebaut werden.

Die Förderprogramme der Europäischen Union führten zu zahlreichen konkreten und für die Bürgerinnen und Bürger sichtbaren Ergebnissen. In vielen Politikfeldern wie der Sicherheit, dem Verkehr, der Bildung, Energie und Gesundheitsfürsorge, der Ausbildung und der Schaffung von Arbeitsplätzen spielt grenzüberschreitende Kooperation eine zentrale Rolle (Zonneveld et al. 2012). Die Europäische Union investiert im Zeitraum von 2014 bis 2020 über $10 \mathrm{Mrd} . €$ in die interregionale Zusammenarbeit, wovon rund $6,6 \mathrm{Mrd} . €$ unmittelbar in die Grenzregionen fließen. Damit soll eine nachhaltige Wirkung der Investitionen sichergestellt werden.

Am Beispiel der bayerisch-tschechischen Grenzregion zeigt sich aber immer wieder, dass trotz der finanziellen Unterstützung viel Potential ungenutzt bleibt. Die faktische Trennung zwischen beiden Ländern, die fast ein Jahrhundert intensiv betrieben wurde, hat sich in die Sozialisation weiter Bevölkerungsteile in der Grenzregion über mehrere Generationen hinweg eingeprägt (Neuss et al. 2012). Für die Weiterentwicklung der Region ist die Schaffung eines gegenseitigen Verständnisses aber von besonderer Bedeu- 
tung, um die Projekte zur Förderung der ländlichen Räume nachhaltig zu verankern. Die politischen Akteure in Bayern und Tschechien verfolgen dazu verschiedene Strategien.

\section{Regionalkooperation Oberpfalz/ Niederbayern und Pilsen}

Lokale politische Entscheidungsträger gelten als Pioniere der grenzüberschreitenden Zusammenarbeit nach dem Ende des Kalten Kriegs (Chilla und Sielker 2018; Gualini 2012). Sie waren es, die Wege gesucht haben, über die historisch gewachsenen und weit verbreiteten Vorurteile und Stereotype aus der Kriegs- und Vertreibungsgeschichte hinweg eine gemeinsame Dialogbasis zu schaffen (Weigl 2008; Novotný 2009). Dies führte dazu, dass heute transnationale Kooperationen auf allen Ebenen des politischen Mehrebenensystems an der Tagesordnung sind.

Durch die besonderen Voraussetzungen der deutschen und tschechischen Staatsorganisation mit einem föderalen System und starken Bundesländern auf der einen Seite und einem stärker zentralistischen System mit der tschechischen Hauptstadt Prag auf der anderen Seite sind verschiedene Formen der Kooperationen möglich. So tritt der Freistaat Bayern im Rahmen der grundgesetzlichen Möglichkeiten quasistaatlich auf und versucht neben der Bundesrepublik Deutschland eigenständige Gesprächskanäle zwischen München und Prag zu etablieren. Die Schaffung einer Repräsentanz des Freistaats Bayerns in Prag im Jahr 2014 unterstreicht diese Bemühungen. Das historisch gewachsene und nie ganz abgelegte Selbstverständnis Bayerns als eigenständiger (Frei-)Staat kann seit den Annäherungen der politischen Entscheidungsträger in München und Prag Anfang der 2010er-Jahre als Vorteil für die grenzüberschreitende Zusammenarbeit verstanden werden. Nun erhalten nämlich auch die Pioniere der transnationalen Kooperation in den Kommunen und Landkreisen die nötige Unterstützung von staatlicher Ebene.

Eine besondere Bedeutung nehmen dabei die (Regierungs-)Bezirke in Bayern und Tschechien ein, die in ihrem Selbstverständnis als zusammengehörige Regionen agieren (Baumann 2006). Ostbayern und Westböhmen wird hier vor allem von den Bezirken Oberpfalz und Niederbayern sowie dem Bezirk Pilsen repräsentiert. Grundsätze und Ziele dieser Zusammenarbeit sind in der gemeinsamen Pilsener Deklaration vom 09. November 2001 niedergelegt. Wesentliche Kooperationsgremien sind die fachlich ausgerichteten Arbeitsgruppen und die jährlich stattfindende Konferenz der regionalen Repräsentanten. Auf dieser Basis wurden die geplanten Regionalkooperationen als Projekt der Europäischen Gemeinschaftsinitiative INTERREG III A angemeldet und paritätisch besetzte Arbeitsgruppen für die wichtigsten Handlungsfelder eingerichtet. Auch hier war die Europäische Union durch ihre Projektförderung wichtiger Impulsgeber für die Regionalkooperation zur Schaffung grenzüberschreitender Netzwerke.

Die Zusammenarbeit wird gegenwärtig vorwiegend durch das Programm zur grenzübergreifenden Zusammenarbeit Freistaat Bayern - Tschechische Republik Ziel ETZ 2014-2020 (INTERREG V) unterstützt. Die Ziele dieser Förderung basieren auf der europäischen Kohäsionspolitik und der langfristigen Strategie Europa 2020 und beinhaltet die Bereiche Forschung, Umweltschutz und Ressourceneffizienz, Bildung und Schaffung von nachhaltigen Netzwerken (Novotný 2018). Seit den 2000er-Jahren wurden mehrere gemeinsame Projekte und Aktivitäten durchgeführt, die den Grenzgebieten sowohl in Ostbayern als auch in Westböhmen zugutekamen. Hierbei fällt auf, dass die meisten dieser Projekte die historischen Entwicklungen des 20. Jahrhunderts aufgriffen und zu integrieren versuchten. Die diskursiven Rahmungen dieser Projekte ließen die geschichtlichen Kontexte und die neuen Ideen der Zusammenarbeit auf der Grundlage aktueller Themen oft unverschränkt nebeneinanderstehen. Die Akteure auf beiden Seiten der Grenze waren dabei stets mit der Frage konfrontiert, wie das gemeinsame historische Erbe in der Region berücksichtigt werden konnte, ohne dabei das Trennende zu stark hervorzuheben, die Vergangenheit dabei aber trotzdem diskursiv mit zentralen Gegenwarts- und Zukunftsthemen zu verbinden. Diese Versuche können mit dem Konzept des Re-Framings analytisch greifbar gemacht werden.

\section{Das Beispiel Drachensee}

Der Drachensee ist ein Beispiel für einen solchen Re-Framing-Prozess. Er ist der Versuch einer Neurahmung einer Grenzlandschaft, die bisher vor allem durch die historischen Transformationsereignisse des 20. Jahrhunderts geprägt ist. Im Rahmen des Re-Framings tritt der kommunale Tourismusverband in Furth im Wald als Organisation auf. Die Figur des Drachen spielt dabei eine zentrale Rolle bei der Vermarktung der Grenzregion. Er übernimmt die Brückenfunktion zwischen der diskursiven Rahmung der Vergangenheit und Zukunft. Der Drache wird als Symbol verwendet, um an den Drachenstich in Furth im Wald, dem ältesten Volksschauspiel Deutschlands, anzuschließen. In der gesamten Region ist der Mythos des Drachen schon seit Jahrhunderten kulturell tief verwurzelt (Baumann 1986). 2018 wurde das Schauspiel in das Bundesweite Verzeichnis Immaterielles Kulturerbe der Deutschen UNESCO-Kommission (2019) aufgenommen (Abb. 1).

Die Erzählung des Drachenstichs basiert auf der mittelalterlichen Hussitenbewegung in Böhmen. Über die Jahrhunderte hinweg wurde es im Kontext aktueller politischer 


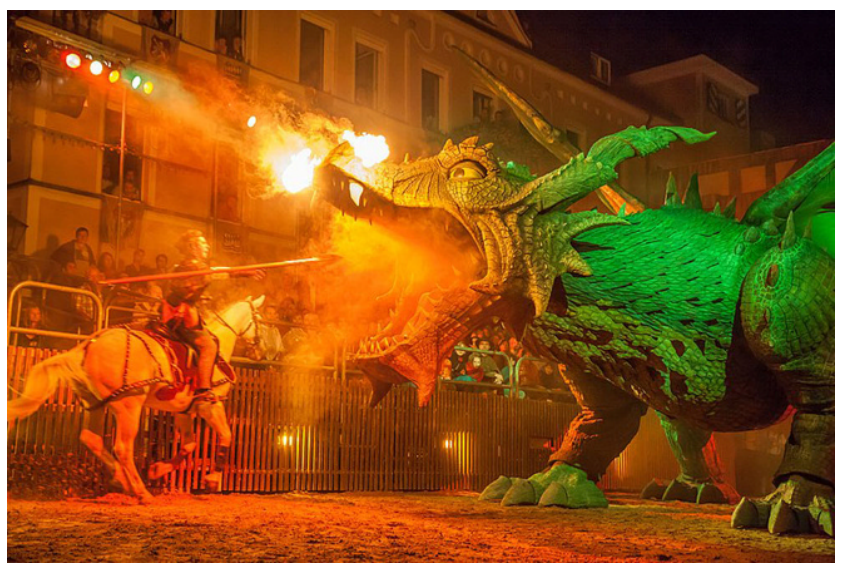

Abb. 1 Der Drachenstich in Furth in Wald. (Quelle: Andreas Mühlbauer, https://de.m.wikipedia.org/wiki/Datei:DerDrachenstich.jpg)

Entwicklungen immer wieder neu interpretiert. Vor dem 1. Weltkrieg wurde das Fest grenzübergreifend gemeinsam gefeiert, mit den Ereignissen ab den 1920er-Jahren entwickelte sich auch im Spiel die bereits beschriebene Distanz zwischen Bayern und Böhmen. Erst nach dem Zusammenbruch des Staatssozialismus in der Tschechoslowakei wurde das Festspiel wieder so ausgerichtet, dass Anknüpfungspunkte für die Menschen aus beiden Ländern möglich waren.

Dass das Symbol des Drachen Kontinuität und Wandel vereint, zeigt ein Blick auf die Drehbücher des Volksschauspiels. Diese wurden im Verlauf der Jahrhunderte immer wieder an aktuelle historische Kontexte angepasst. Beispielhaft dafür steht die Entwicklung des Textes seit dem 2. Weltkrieg: Die Interpretation des Drachenstichs war ausgerichtet am neuen Kalten Krieg und der Drache wurde zum Symbol für die vermeintliche kommunistische Bedrohung aus dem Osten. Durch die Wende in Europa 1989 waren die Darsteller gezwungen, auf die neuen zeitgeschichtlichen Entwicklungen zu reagieren. So entstand in den 2000erJahren erneut eine neue Fassung des Drachenstichs, und nun stand wieder verstärkt das Verbindende zwischen Ost und West im Mittelpunkt, ganz im Sinne des Zusammenwachsens der Grenzregion im Kontext der Europäischen Union. Verbunden wurde mit diesen Neufassungen immer eine Reinterpretation vergangener und gegenwärtiger gesellschaftlicher Zusammenhänge, wobei die Verknüpfung mit konkreten räumlichen Strukturen in der Region stets als Konstante aufrechterhalten wurde. Die raumbezogenen Neurahmungen wurden hauptsächlich auf der diskursiven Ebene vorgenommen, immer aber auch mit räumlichen Phänomenen verbunden.

So ist mit dem Drachenmythos seit 2009 ein staatlicher Hochwasserspeicher verknüpft, der unmittelbar an die Stadt Furth im Wald anschließt. Rund um den 175 ha großen Stauseebereich des Flusses Chamb entstand in den letzten 10 Jahren ein See, der als Freizeit- und Naturerlebnis ökologi- sche und kulturelle Aspekte aufgreift und nicht nur in seiner Wirkung, sondern auch in seiner Planung grenzüberschreitend funktioniert. Grundlage für den Bau des Stausees war bereits ein Beschluss des Bayerischen Landtags vom 15. Juli 1970. Ziel war es schon damals, der strukturschwachen Region um Furth im Wald einen Impuls für die Naherholung zu geben und damit den nötigen Hochwasserschutz für die Stadt und das gesamte Chambtal sicherzustellen. Anknüpfungspunkte an eine mögliche grenzüberschreitende Zusammenarbeit oder gar an eine Neuinterpretation der bisherigen bayerisch-tschechischen Geschichte waren damals nicht angedacht.

Wegen eines langwierigen Rechtsstreits konnte der Freistaat Bayern als Bauherr erst im Jahr 2003 mit dem eigentlichen Dammbau beginnen. Die alte Planung musste dazu sowohl bautechnisch auf den neusten Stand gebracht als auch im Hinblick auf die Gestaltung des Sees völlig überarbeitet werden. Wesentliches Charakteristikum des Sees ist nun die räumliche Trennung in eine Freizeitzone Tiefenwasser und eine Ökozone Flachwasser, die durch eine Inselkette mit Schwimmsteg in der Mitte des Sees gekennzeichnet wird (Abb. 2).

Die Freizeitzone des Sees kann ganzjährig genutzt werden. Durch die unmittelbare Lage am Grenzübergang Furth im Wald kann die Region mit dem Auto über die Bundesstraße 20 erschlossen werden. Damit liegt der Drachensee ungefähr auf der Hälfte der Strecke von Regensburg nach Pilsen. Furth im Wald ist auch mit den öffentlichen Verkehrsmitteln gut erreichbar. Die Länderbahn von Schwandorf nach Prag und die Oberpfalzbahn mit Endhaltestelle Furth im Wald machen eine An- und Abreise im Stundentakt möglich. Der See ist eingebettet in einen größeren regionalen Zusammenhang, der seit einigen Jahren vor allem für das Wandern und Fahrrad- bzw. Mountainbikefahren die Infrastruktur auf der bayerischen und der tschechischen Seite immer mehr ausbaut.

Mit dem Rückgriff auf den Further Drachen erfolgte ein gezieltes Re-Framing der Grenzlandschaft. Der Drache eignet sich deshalb gut als Diskursmedium, weil er in seiner historischen Entwicklung die verschiedenen Transformationsereignisse des 20. Jahrhunderts mit aufgreift, die für die Grenzlandschaft prägend waren. Dadurch, dass der Further Drachenstich aber viel älter ist, kann das Drachensymbol die bisherige Narration der Grenzregion, die sich historisch auf das 20. Jahrhundert bezieht, neu rahmen. Der Drache wird als Symbol der transnationalen Verbindung von Bayern und Tschechen in der Region installiert, der über Jahrhunderte hinweg die Zusammengehörigkeit der Region in den Mittelpunkt stellte. Damit wird das eigentlich Trennende zwischen Bayern und Tschechen, das in den bisherigen Framingprozessen so stark betont wurde, in seiner historischen Dimension erweitert, indem durch die Tradition bis ins Mittelalter die transnationalen Verbindungen in 
Abb. 2 Der Drachensee auf einer Übersichtskarte für Touristinnen und Touristen. (Quelle: Urlaubsland Furth im Wald Hohenbogenwinkel 2017)

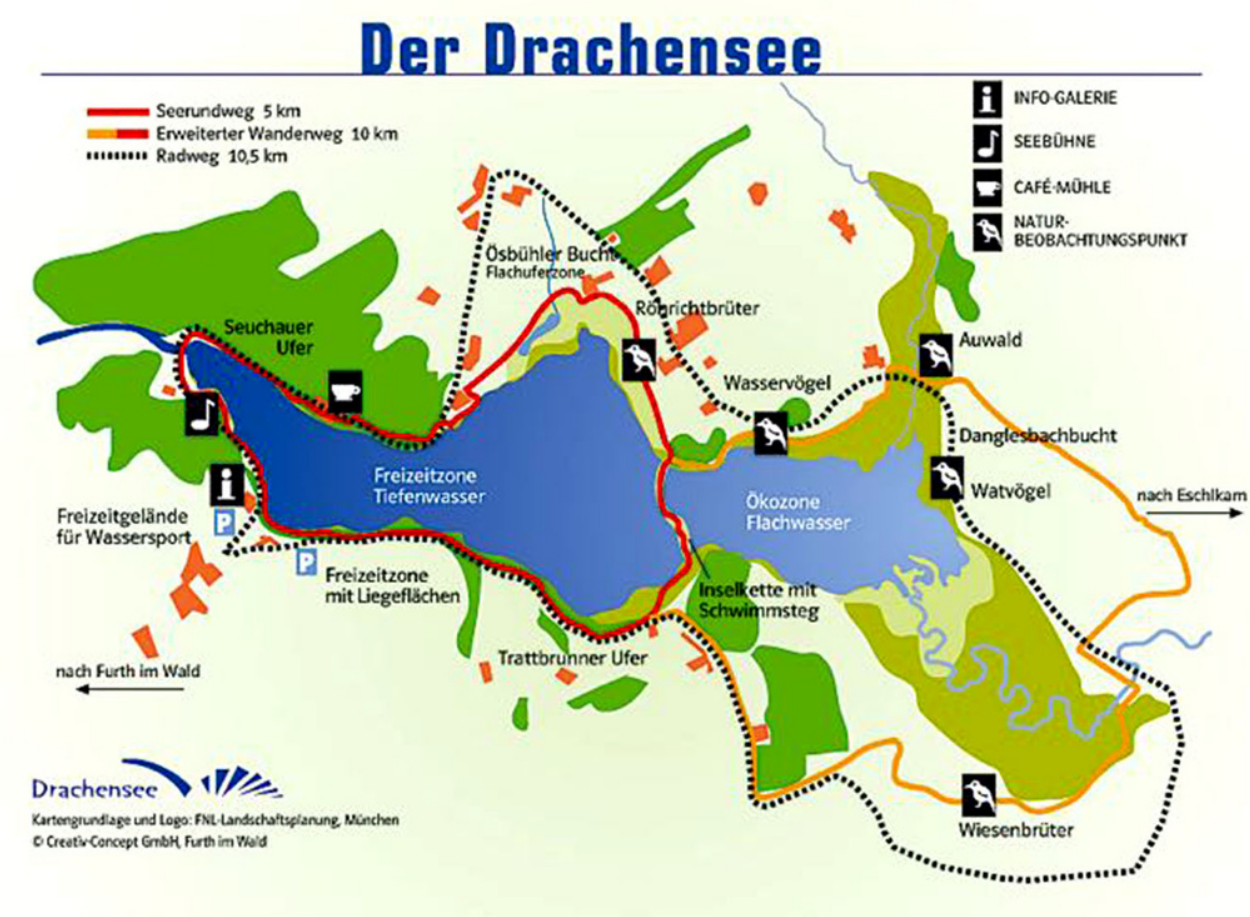

der Region betont werden. Trotzdem folgt damit keine qualitativ-normative Relativierung der Ereignisse des 20. Jahrhunderts, weil das Volksschauspiel Drachenstich in diese Transformationsprozesse ebenfalls integriert war.

Der Drache als Brücke greift also historische Narrationen auf und verbindet sie mit neuen Innovationskonzepten. Denn der Drachensee, der durch das Volksschauspiel seinen Namen erhält, übernimmt selbstverständlich auch noch ganz andere landschaftliche Funktionen. Durch die Verknüpfung mit dem Thema Ökologie und Nachhaltigkeit werden ganz tagesaktuelle Diskurse aufgegriffen und in der Gestaltung der Landschaft umgesetzt. So greifen auch die Informationstafeln rund um den Drachensee, die eine wichtige Funktion im Re-Framingprozess erfüllen, beide Diskursstränge auf: die historische Dimension, symbolisiert durch die Figur des Drachen, und die ökologisch-nachhaltige Dimension, symbolisiert durch die besondere Fokussierung auf naturräumliche Beschreibungen der Landschaft.

Das Re-Framing gelingt insofern, als dass die Besucherinnen und Besucher die Grenzlandschaft als transnationale (und zweisprachige) Grenzlandschaft begreifen, in der Erholung mit der Vermittlung historischer und gegenwartsbezogen verbindender Themensetzung verbunden werden kann. Die Besucher nehmen die Neurahmung der Landschaft an und verbinden mit der Landschaft nun eine Verknüpfung der gemeinsamen Geschichte und der gegenwärtigen Herausforderung der Förderung einer ländlich-peripheren Grenzregion.

\section{Fazit}

Der kommunale Tourismusverband verfolgt die Strategie der touristischen Inwertsetzung einer Grenzlandschaft, die durch ihre ländlich-periphere Lage gekennzeichnet ist. Durch die historische Teilung der Region im Zuge des OstWest-Konflikts konnten bisherige Rahmungen nur national erfolgen. Sie konzentrierten sich auf Ostbayern oder Westböhmen, im jeweiligen politisch-ideologischen System mit entsprechenden Diskursstrategien verbunden. Im Zentrum stand dabei nicht das gemeinsame Herangehen in einer transnationalen Regionalentwicklung, obwohl sowohl die bayerische als auch die tschechische Seite mit ganz ähnlichen Herausforderungen konfrontiert ist.

Durch den Rückgriff auf den Drachen wird nun ein Symbol gewählt, das die gemeinsame Grenzlandschaft diskursiv neu rahmt. Es wird damit das historische Erbe des 20. Jahrhunderts, das von Krieg und Teilung geprägt ist, nicht ausgespart, sondern durch ein gezieltes Re-Framing neu weitergeführt. Die Transformationen des 20. Jahrhunderts werden mit den Narrationen des 21. Jahrhunderts, mit Ökologie und Nachhaltigkeit, verbunden und grenzüberschreitend fokussiert: Nicht nur, indem die organisationale Rahmung sowohl in Bayern als auch in der Tschechischen Republik aufgegriffen wird, sondern auch indem durch eine gezielte grenzüberschreitende Zusammenarbeit im Tourismus eine transnationale, deutsch-tschechische Neurahmung entsteht.

Funding Open Access funding provided by Projekt DEAL. 
Open Access Dieser Artikel wird unter der Creative Commons Namensnennung 4.0 International Lizenz veröffentlicht, welche die Nutzung, Vervielfältigung, Bearbeitung, Verbreitung und Wiedergabe in jeglichem Medium und Format erlaubt, sofern Sie den/die ursprünglichen Autor(en) und die Quelle ordnungsgemäß nennen, einen Link zur Creative Commons Lizenz beifügen und angeben, ob Änderungen vorgenommen wurden.

Die in diesem Artikel enthaltenen Bilder und sonstiges Drittmaterial unterliegen ebenfalls der genannten Creative Commons Lizenz, sofern sich aus der Abbildungslegende nichts anderes ergibt. Sofern das betreffende Material nicht unter der genannten Creative Commons Lizenz steht und die betreffende Handlung nicht nach gesetzlichen Vorschriften erlaubt ist, ist für die oben aufgeführten Weiterverwendungen des Materials die Einwilligung des jeweiligen Rechteinhabers einzuholen.

Weitere Details zur Lizenz entnehmen Sie bitte der Lizenzinformation auf http://creativecommons.org/licenses/by/4.0/deed.de.

\section{Literatur}

Baumann W (1986) Der Drache aus Böhmen - Von der Geschichte zum Festspiel in Furth i. Wald. Bayerische Verlagsgesellschaft, Regensburg

Baumann W (2006) Ein altes Thema - Bayern und Böhmen. Der Landkreis Cham als Vorschlag für die Bayerische Landesausstellung. Beiträge zur Geschichte im Landkreis Cham 23:213-219

Bechtold J, Caesar B, Christmann N, Dorkel N, Evrard E, Heinen S, Morel-Doridat F, Reichert-Schick A (2019) Grenzüberschreitende Raumplanung in der Praxis: Reflexionen zu einem Dialog zwischen Forschung und Praxis in der Großregion. UniGR-CBS Policy Paper, Bd. 1. https://doi.org/10.25353/ubtr-xxxx-fc90-bb02

Chilla T, Sielker F (2018) Grenzüberschreitende Raumentwicklung Bayerns: Ausgangssituation, aktuelle Herausforderungen und konzeptionelle Debatten. In: Chilla T, Sielker F (Hrsg) Grenzüberschreitende Raumentwicklung Bayerns. Dynamik in der Kooperation - Potentiale der Verflechtung. Verlag der ARL, Hannover, S 5-22

Chilla T, Weidinger T (2014) Grenzüberschreitende Zusammenarbeit „Europäische Metropolregion Nürnberg - Westböhmen“

Church A, Reid P (1996) Urban power, international networks and competition: the example of cross-border cooperation. Urban Stud 33:1297-1318. https://doi.org/10.1080/0042098966664

Council of Europe (1980) Europäisches Rahmenübereinkommen über die grenzüberschreitende Zusammenarbeit zwischen Gebietskörperschaften. Amtliche Übersetzung Deutschlands. Sammlung Europäischer Verträge - Nr. 106. https://rm.coe.int/1680078b1d. Zugegriffen: 01. Februar 2020
Deutsche UNESCO-Kommission (2019) Further Drachenstich. https:// www.unesco.de/kultur-und-natur/immaterielles-kulturerbe/imma terielles-kulturerbe-deutschland/bundesweites-18. Zugegriffen: 01. Februar 2020

Fricke C (2014) Spatial governance across borders revisited: organizational forms and spatial planning in metropolitan crossborder regions. Eur Plan Stud 23:849-870. https://doi.org/10. 1080/09654313.2014.887661

Grontmij GmbH (2015) Entwicklungsgutachten für den bayerischtschechischen Grenzraum

Gualini E (2012) Cross-border governance: inventing regions in a Trans-national multi-level polity. disP 152:43-52. https://doi. org/10.1080/02513625.2003.10556833

Havlíček T, Jeřábek M, Dokoupil J (2018) Borders in central Europe after the Schengen agreement. Springer, Cham

Küpper P (2016) Abgrenzung und Typisierung Ländlicher Räume. Thünen Working Paper, Bd. 68

Martin D (2003) „Place-framing“ als place-making: constituting a neighborhood for organizing and activism. Ann Assoc Am Geogr 93:730-750. https://doi.org/10.1111/1467-8306.9303011

Neuss B, Niedobitek M, Novotný L, Rosulek P (2012) Kooperationsbeziehungen in der Europäischen Union - unter besonderer Berücksichtigung des sächsisch-tschechischen Grenzraums. Dr. Kovač, Hamburg

Novotný L (2009) Vergangenheitsdiskurse zwischen Deutschen und Tschechen. Untersuchung zur Perzeption der Geschichte nach 1945. Nomos, Baden-Baden

Novotný L (2018) Grenze und ihr Einfluss auf die regionale Identität und die regionale Entwicklung im deutsch-tschechischen Grenzgebiet. Aussiger Beitr 12:31-48

Svensson S (2015) The bordered world of cross-border cooperation: the determinants of local government contact networks within Euroregions. Reg Fed Stud 25:277-295. https://doi.org/10.1080/ 13597566.2015.1043995

Terlouw K (2012) Border surfers and Euroregions: unplanned crossborder behaviour and planned territorial structures of cross-border governance. Plan Pract Res 27:351-366. https://doi.org/10.1080/ 02697459.2012.670939

Urlaubsland Furth im Wald - Hohenbogenwinkel (2017) Informationsflyer Drachensee. Naturoase, Kulturplatz, Freizeitzone, Ausflugsgebiet

Weigl M (2008) Tschechen und Deutsche als Nachbarn. Spuren der Geschichte in grenzregionalen Identitäten. Nomos, Baden-Baden

Zimmerbauer K (2011) Conceptualizing borders in cross-border regions: case studies of the Barents and ireland-Wales supranational regions. J Borderl Stud 26:211-219. https://doi.org/10.1080/ 08865655.2011 .641322

Zonneveld W, De Vries J, Janssen-Jansen L (2012) European territorial governance. Delft University Press, Delft 